\title{
Localisation des caméras ANPR sur le réseau routier pour le profilage géographique
}

\author{
Marie Trotta $^{1}$, Jean-Paul Donnay ${ }^{2}$ \\ 1. Institut Belge pour la Sécurité Routière, Bruxelles \\ marie.trotta@gmail.com \\ 2. Unité de Géomatique, Université de Liège \\ jp.donnay@ulg.ac.be
}

\begin{abstract}
RÉSUMÉ. Malgré un recours croissant à la technologie des caméras ANPR, en particulier dans les investigations criminelles, la littérature et l'expérience des forces de police avec lesquelles nous avons travaillé, semblent montrer qu'il n'existe pas de méthode systématique afin de localiser le plus adéquatement possible ces installations coûteuses. Partant d'un ensemble de délits localisés, relevant de quatre catégories de vols perpétrés sur une année au sein de la province de Liège (Belgique), nous proposons une méthodologie exploitant, dans une analyse multicritère, la densité de délits, la densité de voirie et la distance entre les sites des caméras. L'approche multicritère permet d'ajuster la pondération des facteurs selon les types de délits. En outre, la méthodologie est itérative, dans la mesure où les sites des caméras sont localisés par phases successives. Un critère de validation des résultats est fourni sous la forme d'un taux de couverture du territoire considéré, qui est fonction du nombre de sites de caméras retenus et du temps de parcours depuis chacun de ces sites. De plus, les conséquences des tentatives d'évitement des sites installés en matière de profilage géographique sont rapidement évoquées.
\end{abstract}

ABSTRACT. Despite increasing use of ANPR camera technology, especially in criminal investigations, literature and the experience of the police with whom we worked, seem to show that there is no systematic method to locate as adequately as possible these expensive facilities. Given a set of four categories of robberies over one year localized within the province of Liège (Belgium), we propose a methodology based on a multi-criteria analysis, making use of density offenses, road density and the distance between the sites of the cameras. Multi-criteria approach can adjust the factor weightings for different types of offenses. In addition, the methodology is iterative, insofar cameras sites are located in successive phases. One result validation criteria is provided in the form of a territorial coverage rate considering the number of selected camera sites and the travel time from each of these sites. Moreover, the consequences for the geographical profiling of attempts to avoid the installed sites are quickly evoked.

MoTS-CLÉS: caméras ANPR, profilage géographique, ingénierie du trafic, estimation à noyaux de densité, analyse multicritère

KEYWORDS: ANPR cameras, geographic profiling, traffic engineering, Kernel Denstiy Estimation (KDE), multi-criteria analysis 


\section{Principes et utilisation des caméras ANPR}

Les caméras dites «ANPR», pour Automatic Number Plate Recognition, constituent un moyen d'identifier les plaques minéralogiques des véhicules en temps réel et, moyennant une connexion à une base de données adéquate, de repérer et éventuellement de reconstituer des portions de trajets de véhicules sur le réseau routier. Leur utilisation a été imaginée dans les années 1990, mais leur banalisation n'est apparue qu'à partir des années 2000 tant pour l'analyse criminelle (PA Consulting, 2004) que pour la gestion du trafic (Friedrich et al., 2008). Il existe des solutions mobiles, les caméras étant installées sur des véhicules, ou des installations fixes sur des poteaux ou des portiques. Une caméra couvre en pratique une voie dans un ou dans deux sens de circulation. Les données qu'elle fournit, géo-localisées et minutées, peuvent être enregistrées à des fins de traitements ultérieurs. Les services de police peuvent ainsi utiliser ces caméras de plusieurs manières, tant en temps réel, qu'en temps différé. Dans ce dernier cas, les données, issues de caméras fixes, sont stockées dans un entrepôt où des applications, de type OLAP et fouille de données, permettent de répondre à des préoccupations décisionnelles, notamment en matière de criminalité (College of Policing, 2013).

C'est dans ce contexte des installations fixes et du traitement de données a posteriori que la problématique de la localisation optimale des caméras ANPR se pose avec le plus d'acuité car il faut, avec un nombre limité d'installations, couvrir le territoire ciblé et innervé par le réseau routier. De plus, pour des questions évidentes de coûts, il est tentant de vouloir exploiter l'infrastructure installée, à la fois pour les besoins de la gestion du trafic et ceux de la recherche criminelle. Or les deux applications ne réclament pas nécessairement les mêmes critères de localisation des caméras.

\section{Un état de l'art lacunaire...}

Si les caméras ANPR font l'objet de publications relativement nombreuses, tant du point de vue technique que dans les médias, la littérature scientifique en la matière est par contre assez rare et très déséquilibrée. Outre les articles strictement techniques, décrivant le matériel et ses caractéristiques de fonctionnement, la plupart des contributions scientifiques portent sur les algorithmes de reconnaissance des caractères alphanumériques des plaques d'immatriculation (Rhead et al., 2012 ; Gurney et al., 2013). La problématique de la localisation des caméras n'est pas abordée explicitement, d'une part parce que bon nombre d'études utilisent des caméras mobiles, et d'autre part parce que dans le contexte de la gestion de trafic, les sites dits «stratégiques" sont identifiés soit de manière empirique (essentiellement selon les comptages routiers et les statistiques d'accidents de la route - PA Consulting, 2004 ; Highways England, 2016), soit sur base des théories de l'ingénierie du trafic (Homburger et al., 2007), éventuellement réduite aux transports publics (Matysiak et al., 2015).

Les instances publiques responsables des infrastructures ne sont pas avares de publications à destination des services techniques. Ainsi en Grande-Bretagne, le 
Home Office a publié des standards nationaux pour les données, l'infrastructure et l'accès aux données des caméras ANPR (Home Office, 2014). En outre, le pays dispose d'un centre national dédié à la gestion et au traitement des données (National ANPR Data Centre) récoltées par plusieurs milliers de caméras établies sur le territoire national. Vis-à-vis du grand public, il n'est pas rare de voir publier sur Internet des listes de caméras localisées (ANPR et autres sans distinction, par exemple Hounslow, 2016), d'autant que leur installation a posé et pose toujours débat quant à la protection de la vie privée (Home Office, 2011). Néanmoins, le rôle croissant des caméras ANPR dans la lutte contre la criminalité - et aujourd'hui le terrorisme - fait que leur nombre et leur localisation précise, et a fortiori les critères prévalant à leur installation, ne sont pas divulgués. Le message est toujours le suivant: "In line with national policy, we do not disclose details of our fixed locations as this information is likely to be of benefit to offenders and if known could reduce the value of ANPR to policing »(Police.UK, 2016). Pour l'anecdote, le même message est repris in extenso sur les sites d'autres pays utilisant la même technologie (par exemple CCTV Dubai, 2015).

La dimension géographique du problème n'est pourtant pas ignorée. La manière dont les caméras ANPR peuvent aider la recherche criminelle est exposée succinctement pour justifier auprès du grand public leur installation et leur densité : «Geographical searches produce a list of VRMs [Vehicle Registration Marks] that have appeared in a particular area, or areas, within specified timeframes. Each geographical area is defined by a centre point and a circle drawn around it using a defined radius. All VRMs that appear at a location, or locations, within the specified timeframe are included in the result set. This search might help to identify associates of an offender, or the vehicles they are known to use. Where more than one vehicle may have been used to leave the scene of an incident, this may help to link a VRM witnessed at the scene to that of an identified associate. " (College of Policing, 2013). Mais une fois encore, les méthodes utilisées pour sélectionner les localisations adéquates ne font l'objet d'aucun commentaire.

\section{Objectif et plan de l'étude}

\subsection{Objectif}

L'objectif poursuivi par cette étude est le positionnement des sites de caméras fixes de surveillance ANPR sur le réseau routier d'un territoire administratif donné. L'opportunité nous en a été donnée par les services du gouverneur de la Province de Liège (Belgique) qui, pour répondre à la demande des polices locales, ont souhaité disposer d'une méthodologie de localisation des caméras ANPR assurant une couverture de l'ensemble du territoire de la province de Liège $\left(3.862 \mathrm{~km}^{2}\right)$. L'installation des caméras ANPR doit permettre de suivre des trajets empruntés par 
les véhicules suspects, d'alimenter les procédures de profilage géographique ${ }^{1}$ exploitées dans la résolution des délits graves et, accessoirement, de participer à la surveillance et la gestion de la circulation routière en général.

Étant donnés le coût et le temps d'installation de telles caméras, la sélection des sites favorables doit en outre être proposée par phases successives, tout en garantissant un taux de couverture croissant (Trotta et Donnay, 2015).

\subsection{Données de l'application}

$\mathrm{Au}$ moment de l'étude ${ }^{2}$, les différentes catégories de vols constituaient la préoccupation majeure des services de police locaux. Bien qu'il ne s'agisse pas du type de délit idéal pour la problématique de la localisation des caméras ANPR (cf. section 4.1), les données étaient disponibles et en nombre suffisant pour tenter l'établissement d'une méthodologie. Les services de police nous ont ainsi fourni les données relatives à quatre types de vols: (1) cambriolages qualifiés dans les habitations, (2) vols à main armée dans les commerces, (3) vols avec violence dans les habitations et (4) vols de véhicules, soit au total 12.353 faits recensés et localisés entre octobre 2013 et 2014 sur le territoire de la province de Liège (table 1).

\begin{tabular}{|l|r|r|r|}
\hline \multicolumn{1}{|c|}{ Type de délits } & $\begin{array}{c}\text { Nombre } \\
\text { de faits } \\
\text { localisés }\end{array}$ & $\begin{array}{c}\text { Nombre } \\
\text { de sites } \\
\text { localisés }\end{array}$ & \% de sites \\
\hline Cambriolages qualifiés dans les habitations & 10.387 & 4.844 & 71.3 \\
\hline Vols à main armée dans les commerces & 150 & 126 & 1,9 \\
\hline Vols avec violence dans les habitations & 121 & 121 & 1,8 \\
\hline Vols de véhicules & 1.695 & 1.694 & 25,0 \\
\hline Total & 12.353 & 6.785 & 100,0 \\
\hline
\end{tabular}

TABLE 1. Nombre de délits par catégories, entre octobre 2103 et octobre 2014

D'autre part, nous avions accès à la base de données du référentiel PICC de la Région wallonne (SPW, 2016), reprenant toutes les informations requises sur le réseau de voirie (à l'exception du trafic) et les adresses postales couvrant le territoire provincial. Cependant, pour une question de confidentialité, la localisation des délits ne nous a été transmise qu'au niveau du nom de rue, et comme plusieurs délits peuvent apparaître par rue, ce sont au total 6.785 «sites » de délits qui ont été géocodés en coordonnées Lambert belge 72 sur les axes de voirie (table 1).

\footnotetext{
1 "Geographic profiling [...] was originally defined as a criminal investigative methodology for analysing the locations of a connected series of crimes in order to determine the most probable area of offender residence" (Rossmo, 2000).

${ }^{2}$ Avant les épisodes dramatiques liés aux activités terroristes sur le territoire belge.
} 
Il ne nous a pas été possible de disposer des adresses postales des auteurs des délits solutionnés, ni des données sur les comptages routiers sur la période considérée, toutes données qui auraient permis d'affiner les valeurs de certains paramètres utilisés dans la méthode. De même, la localisation des portiques déjà installés et équipés de caméras ANPR sur le territoire de la province de Liège et celui des provinces et pays limitrophes, n'a pu être renseignée que de façon très partielle. Ces divers manquements dans les données auraient un impact certain sur les résultats si ceux-ci devaient être considérés du point de vue opérationnel. Cependant, l'étude avait avant tout une portée méthodologique et les données ne sont considérées que comme un cas d'école.

\subsection{Plan}

L'étude est réalisée en trois étapes et incorpore, dans la dernière étape, un processus itératif de sélection des sites où peuvent être installées les caméras :

- L'analyse des zones concentrant un nombre important de délits (hotspots), selon les catégories de délits sélectionnées par les forces de police.

- L'identification des itinéraires susceptibles d'être fréquemment utilisés pour se rendre à / sortir de ces hotspots depuis tout point de la province - sur le réseau routier - et depuis les frontières de la province.

- Le positionnement des sites de caméras sur les tronçons routiers les plus utilisés dans les itinéraires d'accès / sortie des hotspots, en tenant compte de la densité de délits et de la distance entre les sites de caméras. Comme énoncé ci-dessus, ce positionnement doit se faire par phases $(10,20,30,40$ et 50 sites $)$.

\section{Analyse des données criminelles}

\subsection{Types de délits concernés}

Tous les types de délits ne sont évidemment pas susceptibles d'être enregistrés par des caméras ANPR. L'analyse criminelle présuppose, de la part du criminel, un déplacement suffisamment long (en kilomètres et/ou en temps) au moyen d'un véhicule immatriculé de façon reconnaissable. De plus, l'approche repose sur l'une des deux hypothèses suivantes :

- Soit l'identification d'un véhicule à des endroits et des temps déterminés permet de faciliter le traçage d'un véhicule suspect et, finalement, de délimiter une zone de repli vraisemblable du criminel, aussi limitée que possible. En ce sens, l'installation d'un réseau de caméras ANPR est susceptible d'alimenter les procédures de profilage géographique de l'analyse criminelle.

- Soit l'identification récurrente d'un même véhicule dans les environs d'un site criminel et à des moments jugés propices permet de suspecter le véhicule d'avoir participé à la préparation et/ou la réalisation du délit.

Il résulte de ces contraintes et objectifs, que l'efficacité des caméras ANPR dans les investigations criminelles dépend, bien sûr, du nombre de caméras installées, mais 
aussi, d'une part, de la «densité » du réseau routier (connectivité et longueur cumulée sur un territoire de superficie donnée) et, d'autre part, de la propension des criminels à effectuer de longs trajets. Les criminels agissant à proximité de leur lieu de résidence, au comportement dit «maraudeur» (Meaney, 2004 ; Paulsen, 2007), ne sont donc pas la cible idéale des investigations utilisant les caméras ANPR. Or, les cambriolages dans les habitations relèvent surtout de cette catégorie (Snook, 2004), ce qui explique pourquoi le jeu de données sélectionné pour nous par la police ne constitue pas le meilleur échantillon qui soit.

\subsection{Identification des « hotspots " de criminalité}

En première analyse, on pourrait considérer que les endroits où apparaissent de nombreux délits constituent de bons candidats à la localisation des caméras ANPR. En réalité, il ne faut pas se focaliser sur ces endroits eux-mêmes, mais plutôt sur les chemins qui y mènent ou qui permettent de s'en échapper. Il reste que ces « nœuds de concentration des délits » ou hotspots doivent d'abord être identifiés. La technique utilisée en analyse criminelle pour les mettre en évidence est bien maîtrisée, et consiste à transformer le nuage d'évènements ponctuels localisés en une surface continue par un estimateur à noyaux de densité (KDE pour Kernel Denstity Estimation), puis à faire correspondre les sommets de cette surface aux hotspots cherchés (Chainey et al., 2008, Johansson et al., 2015). Le KDE permet d'établir la densité de faits dans un voisinage, lui-même défini par une distance de recherche qualifiée de largeur de la bande passante (figure 1). L'estimateur calcule la densité comme une somme pondérée de faits où chaque poids lié à un fait est inversement proportionnel à la distance séparant la localisation du fait à celle du centre du noyau. La valeur est calculée de proche en proche, par convolution du voisinage dans une grille couvrant l'espace d'étude. La distribution des poids dépend de la fonction de décroissance choisie, tandis que la valeur de densité est également influencée par la taille de la grille spatiale et la largeur de la bande passante.
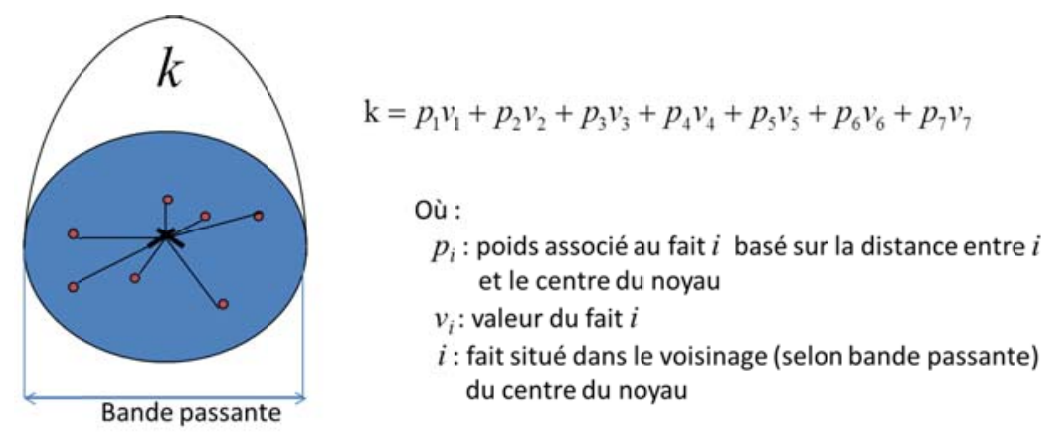

FIGURE 1. Principe du calcul d'une densité de noyau (fonction gaussienne) 
Ce dernier paramètre est jugé important par la littérature (Chainey, 2013) et requiert dès lors un calibrage approprié selon la thématique analysée. Dans cette étude, nous postulons que la bande passante ne peut être supérieure à la distance moyenne parcourue sur le réseau routier par les criminels, afin d'être en mesure de discerner des hotspots associés à différentes origines.

Ces valeurs varient fortement d'un territoire à l'autre mais sont générallement de l'ordre de $6 \mathrm{~km}$ pour les délits considérés (Vandeviver et al., 2015). La distribution des distances étant fortement asymétrique, l'examen de plusieurs paramètres de position est utile (moyenne et médiane). Nous avons procédé à une estimation des distances parcourues par les auteurs des faits sur base des statistiques des faits résolus fournies par la police (nous n'avons pas eu accès au détail des données, mais seulement à des valeurs statistiques calculées à notre demande). On notera tout d'abord que le pourcentage de faits résolus $(8,2 \%)$ est faible et pourrait ne pas être représentatif de l'ensemble des comportements. En effet, une origine plus lointaine de l'auteur pourrait être associée à une difficulté additionnelle de résolution de l'enquête.

Pour l'échantillon de faits retenu par la police, la distance moyenne parcourue par les auteurs est de $6,7 \mathrm{~km}$ et la valeur médiane se situe dans la classe 4 à $6 \mathrm{~km}$ (figure 2). Conformément à ce que propose la littérature, la bande passante choisie de $2,5 \mathrm{~km}$ est inférieure à ces deux valeurs. Les distances moyennes pour chaque type de faits sont supérieures à $5 \mathrm{~km}$ et les distances médianes supérieures à $2,5 \mathrm{~km}$. Cependant, une incertitude persiste à propos des cambriolages dans les habitations, qui représentent la plus grande proportion de faits $(>70 \%)$. Leur valeur médiane est comprise dans la catégorie de distances de 2 à $4 \mathrm{~km}$, c'est-à-dire du même ordre de grandeur que la bande passante choisie. Cette situation limite n'est pas pénalisante pour la suite de la procédure, mais illustre bien le caractère circonscrit de ce type de délits et, par voie de conséquences, sa faible adéquation avec les techniques d'investigation utilisant des caméras ANPR. Le KDE utilisant une fonction gaussienne est calculé avec cette bande passante de 2,5 km sur les éléments linéaires du réseau de voirie (KDE linéaire utilisant les distances le long du réseau) préalablement rastérisés à une résolution de $30 \mathrm{~m}$.

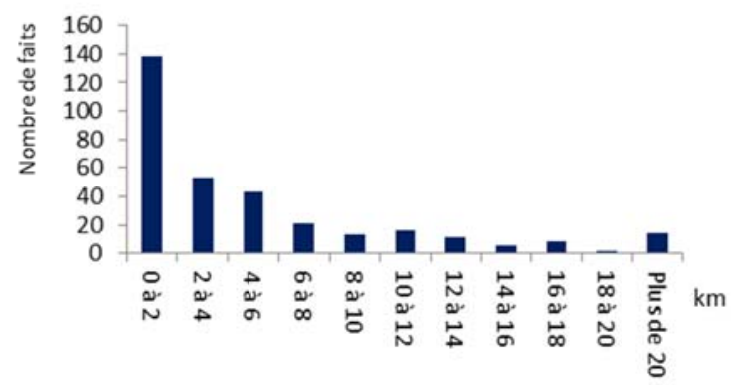

FIGURE 2. Répartition des distances parcourues par les auteurs de cambriolages 
Les quatre surfaces de densité ainsi engendrées présentent une grande stabilité quant à la position de leur maxima. Cela s'explique aisément par la nature des délits considérés qui, en dernière analyse, sont largement corrélés à la densité de population. Ceci nous a incités à construire une surface de densité globale, prenant en compte tous les faits localisés avec les mêmes paramètres, mais non limitée cette fois aux seuls éléments linéaires. . La surface d'accessibilité construite par KDE est une surface continue sur laquelle des inversions de pentes peuvent être interprétées comme participant aux limites des aires d'influence des hotspots. En exploitant cette propriété, il est possible d'extraire automatiquement ces aires selon l'algorithme d'extraction de « bassins versants », après avoir inversé la surface d'accessibilité et fixé une limite d'extension aux «bassins» (ici $8 \mathrm{~km}$ afin d'assurer la couverture complète du territoire). Le minimum de chaque «bassin » ainsi identifié correspond à un hotspot.. 37 hotspots sont ainsi identifiés, concentrés dans les parties les plus densément peuplées de la province et situés à proximité immédiate des voies rapides (figure 3).

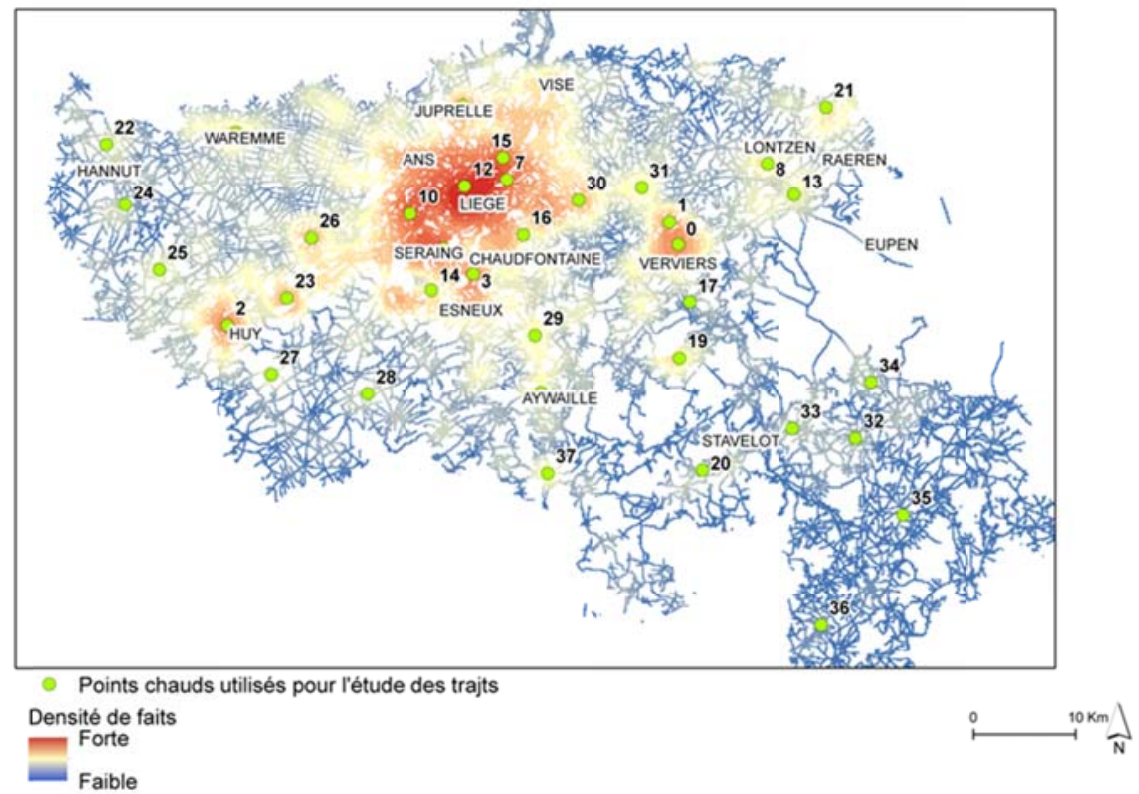

FIGURE 3. Zones de concentration et identification des points chauds (hotspots) pour tous les types de délits sur la province de Liège

\section{Identification des plus courts chemins vers les hotspots}

L'étape suivante consiste à repérer les tronçons et nœuds du réseau fortement empruntés pour se rendre vers les hotspots. Il faudrait pour cela envisager tous les chemins possibles depuis les origines, en nombre limité (les hotspots), vers toutes les destinations possibles au sein de la province (tous les croisements du réseau 
routier). Pour limiter la complexité du problème, seuls les chemins les plus courts, entre origines et destinations, ont été considérés ${ }^{3}$. De plus, en l'absence de données précises qui auraient permis l'application des méthodes de l'ingénierie du trafic ${ }^{4}$, seuls sont pris en compte pour la construction des plus courts chemins, la topologie du réseau, le sens de circulation permis sur l'axe de voirie et la vitesse maximale autorisée par tronçon.

Afin de générer les plus courts chemins depuis chaque nœud (croisement) de la voirie, l'algorithme de « recherche les ressources les plus proches » est utilisé. "Cet algorithme recherche une ou plusieurs ressources les plus proches d'un incident d'après le temps de trajet, la distance et autres coûts, et génère en sortie le meilleur itinéraire, la ressource choisie et les directions entre l'incident et la ressource. » (ESRI, 2015). Conformément à ce qui est décrit ci-dessus, l'algorithme de Dijkstra (1959) utilisé dans cette application conduit au calcul de la route directe exacte et n'exploite pas l'option de réseau hiérarchisé (ESRI, 2005).

Le hotspot est ici considéré comme l'unique ressource (origine), et chaque nœud comme un incident (destination). Le temps de trajet sur chaque tronçon (arc entre 2 nœuds) est calculé d'après la vitesse maximale autorisée sur celui-ci. Le temps de traitement étant dépendant du nombre de destinations, celles-ci ont été réduites de manière à ce que les nœuds soient au moins distants entre eux de $100 \mathrm{~m}$, ce qui laisse quand même. 50.590 destinations à prendre en considération. Les plus courts chemins vers toutes les destinations sont ainsi construits au départ des 37 hotspots identifiés à l'étape précédente.

La fréquence d'utilisation de chaque tronçon, soit le nombre de fois qu'un tronçon participe à un plus court chemin, est ensuite calculée vis-à-vis de chaque hotspot (figure 4). Le processus est réalisé en mode maillé à une résolution de $30 \mathrm{~m}$, et il met en évidence les goulots d'étranglements (passages obligés) du réseau routier. Les inévitables effets de frontière d'un territoire limité induisent également une plus forte fréquence d'utilisation des nœuds en situation centrale, mais d'autant moins perturbante que le territoire est étendu et la densité de voirie élevée. La densité globale des plus courts chemins vers tous les hotspots est obtenue par sommation entre pixels homologues des 37 images de densités calculées à l'étape précédente. Comme seules les routes avec un trafic important sont en principe candidates à un site ANPR, seuls les tronçons dont la valeur de densité globale est supérieure à un seuil sont sélectionnés. Le choix de la valeur seuil est arbitraire mais doit assurer la mise en évidence des axes innervant toute la province, en dehors des autoroutes (aux valeurs de densité systématiquement très élevées) et en particulier dans les parties périphériques de la province. La valeur seuil de 90 a été choisie en tenant compte de ces considérations.

\footnotetext{
${ }^{3}$ Sur un territoire plus limité et strictement urbain, la prise en compte d'un indice de redondance sur les chemins les plus courts devrait être envisagé (Sevtsuk \& Mekonnen, 2012).

${ }^{4}$ Pour rappel, aucune donnée de trafic n'a pu être obtenue pour réaliser cette étude.
} 


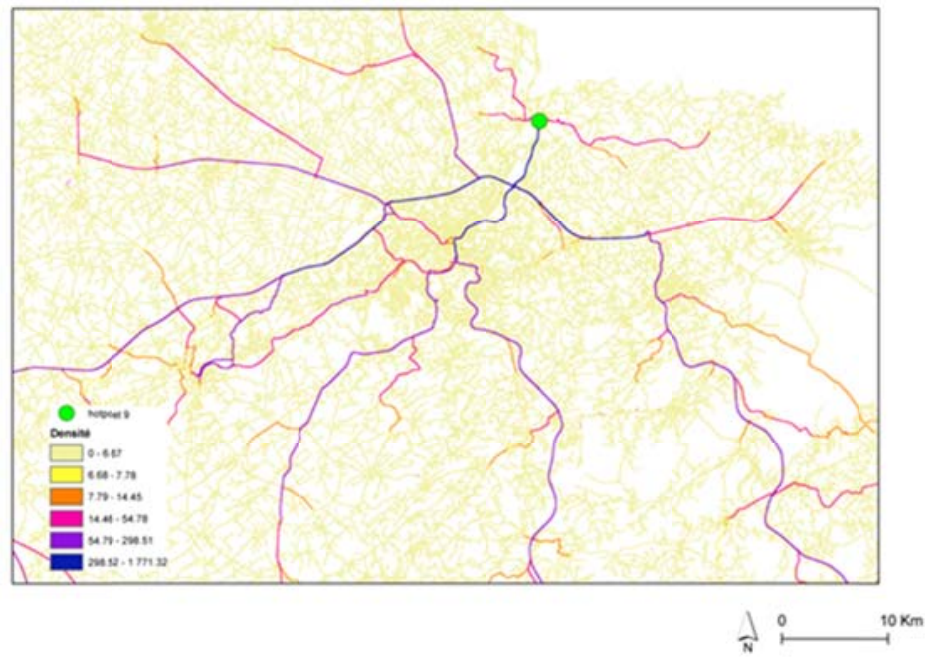

FIGURE 4. Densité de plus courts chemins vers un hotspot. Les autoroutes sont logiquement mises en évidence mais aussi des tronçons à vitesse plus faible ne pouvant être identifiés a priori

\section{Positionnement des sites ANPR}

La sélection des sites de caméras doit être opérée de façon itérative, par groupes de 10 sites, jusqu'à un total de 50 sites, tout en conservant à chaque étape les sites préalablement sélectionnés. Ceci doit permettre d'échelonner dans le temps l'installation de l'infrastructure, tout en améliorant au fur et à mesure son efficacité. Rappelons qu'un «site ANPR » peut accueillir une ou plusieurs caméras ANPR selon la configuration de la voirie et les capacités techniques des caméras utilisées.

Les dix premiers sites ont été positionnés sur les autoroutes à l'entrée de la province (8), ainsi que sur les deux localisations présentant les plus fortes densités de plus courts chemins situées respectivement aux abords des deux agglomérations principales de la province (Liège et Verviers).

La sélection des sites suivants, par groupe de 10, repose sur la valeur d'un indice multicritère calculé en chaque pixel de la voirie. L'analyse multicritère reprend deux facteurs préalablement calculés : la valeur de la densité globale de délits obitenue par KDE, et la densité globale des plus courts chemins vers tous les hotspots. Ce dernier facteur reste contraint par la valeur seuil de 90 discutée ci-dessus. Un troisième facteur est introduit dans l'analyse pour tenir compte de la distance aux sites déjà sélectionnés aux itérations précédentes. La méthode d'agrégation multicritère est la plus simple (équation 1), dans la mesure où elle facilite l'interprétation des scénarios utilisant des facteurs et des poids distincts. Les facteurs sont préalablement centrés- 
réduits et ramenés sur une échelle commune codée sur un octet, tandis que ramenés sur un même intervalle et leurs poids sont normalisés (somme des poids unitaire).

$$
I C=\left(\sum_{i=1}^{n} p_{i} F_{i}\right) \prod_{j=1}^{m} C_{j}
$$

où :

$$
\begin{array}{ll}
I C: \text { indice multicritère } & p: \text { poids des facteurs } \\
F: \text { contributions des facteurs } & C: \text { valeurs (binaires) des contraintes }
\end{array}
$$

L'ambiguïté des objectifs au moment de réaliser cette étude préalable (profilage géographique ou gestion de trafic; vols de différentes catégories ou grand banditisme...) nous a conduit à privilégier une approche permettant de traduire et de modifier aisément la structure de préférences des acteurs. Les valeurs des poids présentées au tableau 2 sont déterminées selon une procédure analytique hiérarchique (AHP ; Saaty, 1980), en fonction de l'expérience de l'équipe (police et chercheurs) travaillant sur l'étude dans le domaine du profilage géographique (Trotta \& Donnay, 2015). Les méthodes relevant de ce domaine donnent une place prépondérante à la distance et à sa décroissance pour définir les zones prioritaires de recherche d'un criminel. Il semblait dès lors judicieux d'attribuer un poids plus important aux critères de distance. Le premier critère, la distance entre caméras, assure une couverture de l'ensemble du territoire. Le deuxième critère garantit une préférence pour les tronçons fortement utilisés sur la province. Il faut toutefois noter que ces tronçons sont principalement situés au niveau des autoroutes et que les auteurs peuvent mettre en place des stratégies d'évitement. Le poids associé à ce second critère est dès lors moins important que celui affecté au critère de distance entre caméras. Le troisième critère favorise un positionnement des caméras à proximité des hotspots. Ici encore, sa pondération faible est dictée par les théories du profilage géographique. En effet, s'il est essentiel d'avoir un positionnement des caméras couvrant les zones problématiques, par définition les hotspots peuvent attirer les criminels sur des distances importantes de par l'attractivité des cibles potentielles qui y sont concentrées. Il est dès lors souhaitable de limiter le poids associé à ce facteur.

\begin{tabular}{|l|c|}
\hline Critères & Poids des facteurs \\
\hline F1 : distance aux caméras positionnées & P1:0,692 \\
\hline F2 : densité des plus courts chemins & P2: 0,231 \\
\hline F3 : valeur de la densité globale du KDE & P3: 0,077 \\
\hline $\mathrm{C}_{1}:$ densité des plus courts chemins supérieure à 90 \\
\hline
\end{tabular}

TABLE 2. Facteurs et contrainte de l'analyse multicritère 

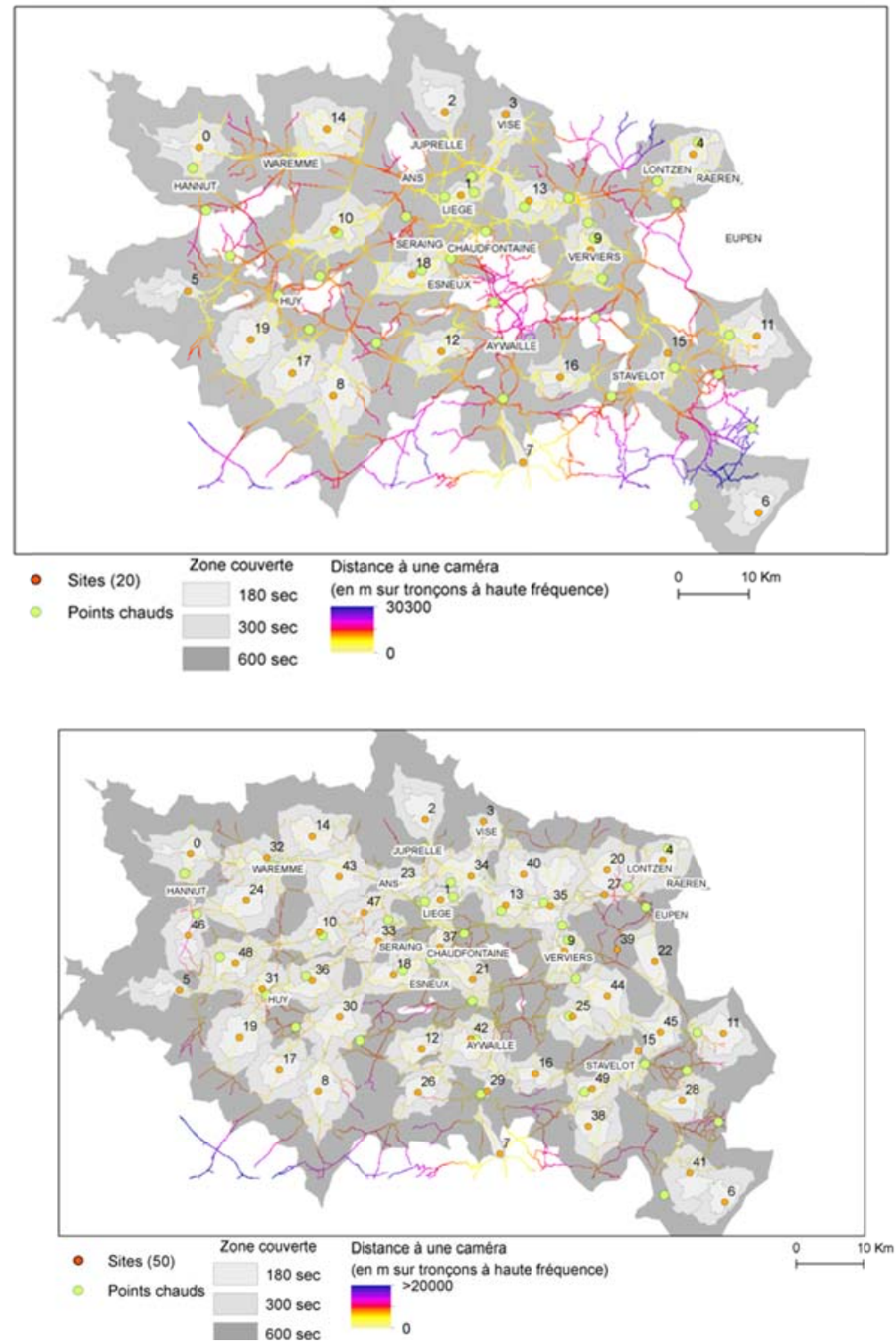

FIGURE 5. Positionnement des sites ANPR et couverture par temps de parcours (20 sites en haut, 50 sites en bas) 
Pour rappel, le vecteur des poids correspond au premier vecteur propre de la matrice d'inertie formée par les comparaisons de l'importance relative des facteurs deux à deux (importance du facteur en ligne vs. du facteur en colonne). Les importances relatives sont établies sur une échelle de 9 paliers (4 de part et d'autre de l'équivalence) et fixées par les acteurs de l'étude. Il est clair que la procédure reste arbitraire et qu'elle pourrait donner lieu à une étude de sensibilité. Mais il faudrait pour ce faire disposer de données complémentaires, notamment relatives au comportement des auteurs de délits afin de quantifier par exemple les phénomènes d'évitement. D'un point de vue pratique, le calcul des poids peut être implémenté dans n'importe quel tableur ou logiciel statistique, tandis que le calcul des valeurs de l'indice est réalisé au moyen des fonctions d'algèbre de cartes d'un quelconque logiciel SIG capable de traiter des données maillées. La totalité de la procédure est disponible parmi les fonctionnalités d'aide à la décision spatiale de plusieurs logiciels (ex. ArcGIS, Idrisi, QGIS, etc.).

Les sites sont donc positionnés d'après les résultats de l'analyse multicritères, en sélectionnant les 10 sites présentant les meilleures valeurs d'indice, et en régénérant le facteur de distance à chacune des itérations. Sont ainsi déterminés les 20, 30, 40 et 50 sites les plus appropriés (figure 5).

\section{Validation des résultats}

En l'absence de caméras installées, et ne pouvant accéder aux données détaillées sur les délits résolus, il ne nous a évidemment pas été possible de valider la proposition de localisation des sites des caméras sur base de cas observés. L'objectif principal qui nous était assigné (cf. $\S 3.1$ ) et qui peut faire l'objet d'une tentative de validation porte sur la couverture du territoire assurée par les sites proposés. Par conséquent, à titre de validation de la procédure, un taux de couverture des hotspots selon le temps de parcours depuis un site ANPR a été calculé et classé en différents pas de temps. Ce taux correspond au ratio de la somme des densités couvertes pour le pas de temps donné, divisé par la somme des densités de faits sur la totalité du territoire de la province. On remarque que les couvertures varient substantiellement pour les temps de parcours de 3 à 5 min (figure 6). Avec un pas de temps de 10 minutes, on constate que la totalité de la province est couverte dès l'installation de seulement 20 sites ANPR sur le territoire.

Les distances maximale et moyenne à un site ANPR diminuent progressivement à chaque itération de la procédure de sélection des sites (figure 7): de respectivement $36 \mathrm{~km}$ et $19 \mathrm{~km}$ (pour 20 sites) à moins de $20 \mathrm{~km}$ et de $10 \mathrm{~km}$ (pour 50 sites). Ces distances restent toutefois plus élevées que les distances moyennes parcourues par les malfaiteurs pour les types de délits envisagés ici (cf. figure 2). 


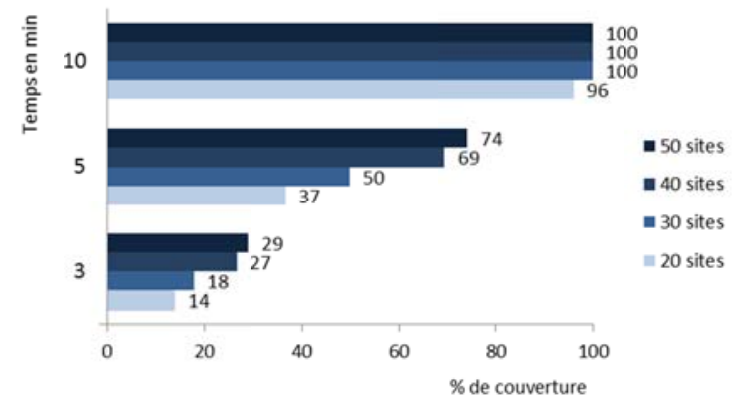

FIGURE 6. Couverture des hotspots selon le temps de trajet depuis un site ANPR

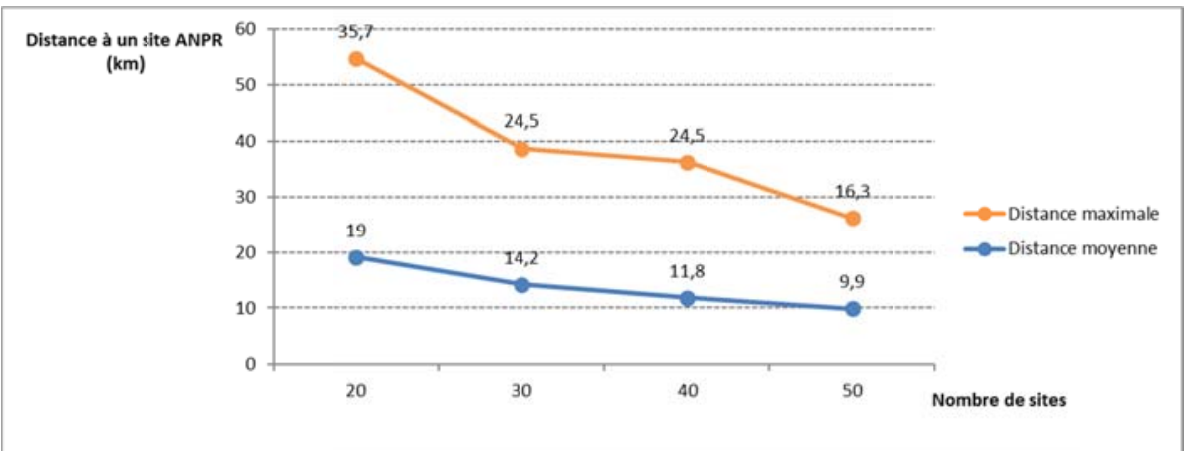

FIGURE 7. Distances maximum et moyenne au site de caméras ANPR le plus proche, selon les scénarios de 20 à 50 sites

Une autre dimension de la problématique qui peut faire l'objet d'une vérification dans le cadre de cette procédure, porte sur le caractère dissuasif de l'implantation de caméras sur les velléités de délits. Les sites d'implantation restant en nombre limité, leur localisation sera vraisemblablement connue par le milieu criminel. On peut donc s'attendre à ce que les malfaiteurs recourent à des itinéraires d'évitement pour atteindre / quitter les hotspots, itinéraires nécessairement plus longs que les plus courts chemins le long desquels sont implantées les caméras. Or, selon la théorie du choix rationnel utilisée en criminologie, le comportement spatial des criminels implique le principe du moindre effort, qui se traduit notamment par la réduction des déplacements autant que faire se peut, tout en restant en dehors de la zone-tampon entourant leur point d'attache (Brantingham \& Brantingham, 1990). Si l'agencement des sites d'implantation des caméras est tel que les temps de parcours sont sensiblement augmentés par les itinéraires d'évitement, on peut logiquement s'attendre à une moindre attractivité des hotspots.

Afin de tester cette hypothèse, nous avons recalculé les temps de trajets entre les hotspots et les nœuds du réseau routier (cf. section 5), mais en supprimant du réseau 
les tronçons où sont localisés les sites ANPR. La distance-temps sur les tronçons est toujours basée sur les vitesses maximales autorisées, tandis que des «barrières » sont introduites sur les tronçons porteurs de caméras afin d'en interdire l'usage dans les parcours. La situation a été examinée dans deux cas de figure : avec 20 et avec 50 sites ANPR. Les temps de parcours, en secondes, ont été comparés à ceux obtenus initialement (absence de barrières) par simple soustraction entre couches maillées («algèbre de cartes ») et sont présentés dans le tableau 3.

\begin{tabular}{|c|c|c|c|c|c|}
\hline Temps (s) & $\mathbf{0}$ & $\mathbf{2 0}$ & $\mathbf{5 0}$ & $\boldsymbol{\Delta} \mathbf{0 - 2 0}$ & $\boldsymbol{\Delta} \mathbf{0 - 5 0}$ \\
\hline Moyen & 2020 & 2093 & 2218 & 73 & 198 \\
\hline Maximum & 5893 & 6107 & 7259 & 2100 & 4372 \\
\hline
\end{tabular}

TABLE 3. Temps de parcours, en secondes, vers les hotspots depuis les nouds du réseau routier

0 : pas d'évitement ; 20 : évitements des tronçons dans le cas de 20 caméras ; 50 : évitements des tronçons dans le cas de 50 caméras

Les différences moyennes de temps de parcours induites par l'évitement des sites de caméras paraissent limitées : de l'ordre de 1 à 3 minutes dans les cas évoqués de 20 et 50 sites ANPR. On pourrait dès lors craindre un délai trop faible pour avoir un effet de dissuasion important. Cependant, vis-à-vis des distances parcourues pour les types de délits envisagés (cf. figure 2) et des vitesses autorisées sur la plus grande partie du réseau routier (maximum $90 \mathrm{~km} / \mathrm{h}$ hors autoroutes), ces délais supplémentaires, aussi petits soient-ils, augmentent très significativement les temps de parcours relatifs.

D'autre part, les écarts entre temps de parcours maxima peuvent s'avérer assez considérables dans les situations extrêmes (près d'une heure et quart de retardement). De tels écarts n'apparaissent pourtant que pour les trajets les plus longs, traversant la province de part en part, donc moins vraisemblables pour les types de délits envisagés dans cette analyse.

Ces différences peuvent ensuite être visualisées pour chaque hotspot comme l'illustre la figure 8. Dans celle-ci, quatre hotspots $\left(\mathrm{n}^{\circ} 12,21,22,36\right)$ ont été sélectionnés en des endroits très différents de la province. On peut clairement constater une augmentation des temps de parcours, allant localement jusqu'à un facteur 10, entre la simulation d'évitement de 20 sites de caméras (à gauche) et celle évitant 50 sites (à droite).

De plus, la carte met en évidence une très nette variation spatiale de l'augmentation du temps de parcours. En principe, les augmentations les plus importantes réduisent d'autant la probabilité qu'un délinquant se rende au hotspot correspondant pour commettre un délit. À titre d'exemple, dans le cas du hotspot 36 l'augmentation des temps de parcours provoquée par l'évitement des 50 caméras (en 
bas à droite de la figure 8) rend peu probable une origine située à l'est de la province. Ce type d'interprétation peut d'ailleurs être exploité dans le cadre des analyses de profilage géographique, afin de limiter l'aire d'investigation et de concentrer les ressources de l'enquête sur les zones les plus plausibles.
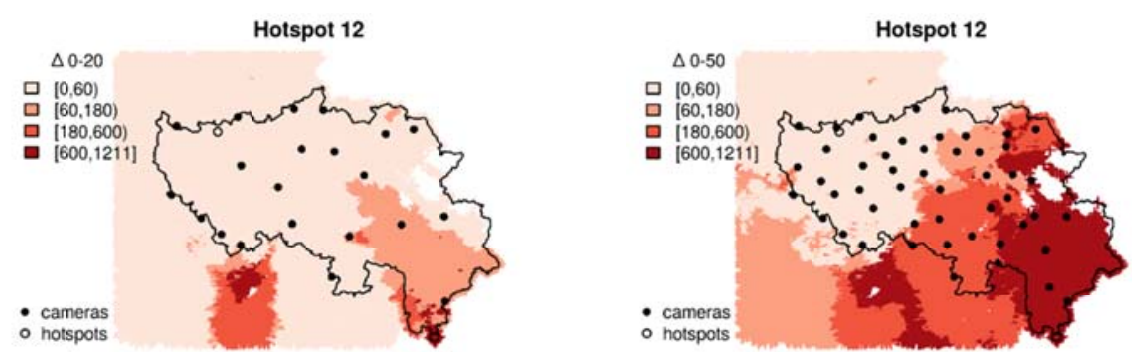

Hotspot 21
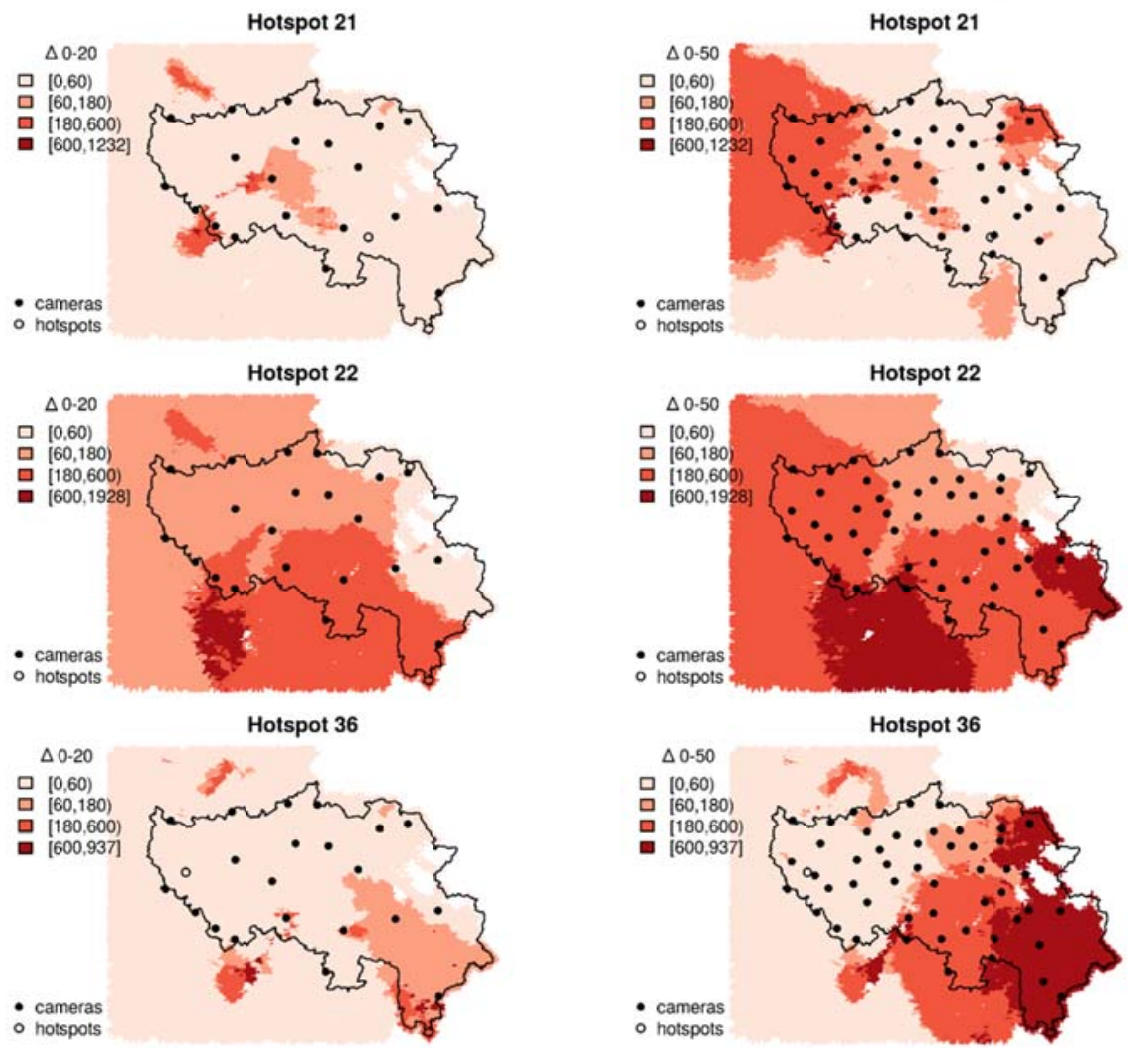

FIGURE 8. Augmentation des temps de parcours pour rejoindre les hotspots 12, 21, 22 et 36, depuis les nœuds du réseau routier, provoquée par l'évitement de 20 et 50 sites de caméras 


\section{Conclusions}

L'absence de méthode avérée pour localiser les caméras ANPR le long du réseau routier nous a amené à proposer une approche combinant des outils classiques de la cartographie criminelle (KDE), de l'ingénierie du trafic (plus courts chemins) et de l'aide à la décision spatiale (analyse multicritère). Vis-à-vis des données traitées, relatives à différentes catégories de vols, le recours aux caméras ANPR n'est utile que dans le cas de bandes organisées parcourant de grandes distances. Mais le canevas méthodologique reste cohérent, quel que soit la nature des délits étudiés.

Les quelques biais qu'il a fallu consentir, dus principalement aux données et à la part d'arbitraire inhérente aux méthodes, constituent autant de pistes de recherche pour une amélioration de l'approche dans un but opérationnel. Ainsi, des données complémentaires et/ou plus précises devraient permettre d'affiner des paramètres importants, tels que la largeur de la bande passante et la fonction de lissage du KDE, ou les vitesses maximales praticables sur le réseau routier en fonction du trafic et de la période. Elles devraient aussi permettre de valider la méthode sur des cas de délits résolus en comparant les localisations des caméras proposées avec les parcours effectivement empruntés par les criminels. En outre, le modèle multicritère offre une grande souplesse d'adaptation dans la sélection des critères et de la fonction d'agrégation s'il fallait, par exemple, intégrer des délits de natures très différentes.

Enfin, l'augmentation des temps de parcours induite par l'évitement des tronçons routiers porteurs de caméras ANPR, telle qu'évoquée dans la validation, pourrait aussi profiter de valeurs plus précises de vitesses. Dans ce cas, l'analyse multicritère pourrait utilement faire usage de la possibilité d'évitement des sites ANPR.

C'est donc aux services de police, à qui la procédure a été présentée, qu'il revient d'affiner la proposition sur base des données détaillées qu'ils possèdent et de procéder à diverses simulations en jouant sur les paramètres du modèle multicritère.

À l'heure où les plans d'équipement de caméras ANPR se multiplient, dans le cadre d'une politique anti-terrorisme, une réflexion qui incorpore la dimension spatiale nous paraît s'imposer.

\section{Références}

Brantingham P.J. \& Brantingham P.L. (1990). Environmental Criminology. Waveland Press, Long Grove (Ill.).

CCTV Dubai (2015). ANPR Camera Dubai. http://cctvdubai.biz/anpr-camera-dubai/ Consulté le $21 / 06 / 2016$.

Chainey S. (2013) Examining the influence of cell size on kernel density estimation crime hotspots maps for predicting spatial patterns of crimes, Bulletin de la Société géographique de Liège, 60, p.7-19.

Chainey S. Tompson L. Uhlig S. (2008) The Utility of Hotspot Mapping for Predicting Spatial Patterns of Crime, Security Journal, 21 (1-2), p. 4-28. 
College of Policing (2013). Authorized Professional Practice - Investigations Investigative Strategies - ANPR, Londres. https://www.app.college.police.uk/appcontent/investigations/investigative-strategies/investigative-strategiesanpr/ Consulté le 21/06/2016.

Dijkstra, E.W. (1959) A note on two problems in connection with graphs, Numero Math, 1, p. 269-271.

ESRI (2005) Hierarchical Routes in ArcGIS Network Analyst, An ESRI White Paper - July 2005, Redlands.

ESRI (2015). ArcGIS Resources. http://resources.arcgis.com/fr/help/main/10.2/index.html\#//004800000028000000 Consulté le 25 mai 2015.

Friedrich, M., Jehlicka, P. \& Schlaich, J. (2008). Automatic Number Plate Recognition for the Observance of Travel Behaviour, $8^{\text {th }}$ International Conference on Survey Methods in Transport, p. 17.

Gurney, R. Rhead, M., Lyons, V. \& Ramalingam, S. (2013) The effect of ANPR Camera Settings on System Performance (poster), IET $5^{\text {th }}$ ICDP Conference Proceedings on Imaging for Crime Prevention and Detection, Londres.

Highways England (2016). Cameras - Fair Processing Notice. http://www.highways.gov.uk/traffic-information/traffic-informationservices/traffic-cameras/cameras-fair-processing-notice/ consulté le 20/06/2016.

Homburger W.S., Hall J.W., Reilly W.R., Sullivan E.C. (2007). Fundamentals of traffic engineering (16th edition), Institute of Transportation Studies, University of California, Berkekey.

Home Office (2011). Consultation on a code of practice relative to surveillance cameras, Londres.

https://www.gov.uk/government/uploads/system/uploads/attachment_data/file/517 367/code-surveillance-cameras.pdf Consulté le 21/06/2016.

Home Office (2014). National ANPR Standards for Policing: Part 1 - Data Standards; Part 2 - Infrastructure Standards; Part 3 -Data Access and Management $\begin{array}{lllll}\text { Standards, } & \text { p. } & 11, & 21, & 16 .\end{array}$ http://www.npcc.police.uk/FreedomofInformation/ANPR.aspx Consulté le 21/06/2016.

Hounslow (2016). List of CCTV locations. London Borough of Hounslow. http://www.hounslow.gov.uk/cameralistsnew30_june_2016_pdf. Consulté le $21 / 06 / 2016$

Johansson E., Gahlin C., Borg A. (2015). Crime Hotspots: An Evaluation of the KDE Spatial Mapping Technique, Proceedings of the 2015 European Intelligence and Security Informatics Conference (EISIC), p. 69-74.

Matysiak, A., Kruszewski, M. Niezgoda, M. \& Kaminski, T. (2015) The analysis of ANPR camera location points in bus lanes monitoring system in the city of Warsaw, Journal of KONES, 20(4), p. 269-275.

Meaney, R. (2004). Commuters and Marauders: An Examination of the Spatial Behaviour of Serial Criminals, Australia, Journal of Investigative Psychology and Offender Profiling, p. 121-137. 
PA Consulting (2004) Driving crime down. Denying criminals the use of the road, Report study commissioned by the Home Office Police Standards Unit (PSU), p. 170.

Paulsen D. (2007). Improving Geographic Profiling through Commuter/Marauder Prediction, Police Practice and Research, 8(4), p. 347-357.

Police.UK (2016). How police forces and other law enforcement agencies use Automatic Number Plate Recognition (ANPR). https://www.police.uk/information-andadvice/automatic-number-plate-recognition/ Consulté le 21/06/2016.

Rhead, M., Gurney, R., Ramalingan, S. \& Cohen, N. (2012) Accuracy of Automatic Number Plate Recognition (ANPR) and Real World UK Number Plate Problems, Proceedings $46^{\text {th }}$ IEEE International Conference on Security Technology, p. 286291.

Rossmo (2000). Geographic Profiling. Boca Raton, CRC Press.

SPW (2016). Géoportail de Wallonie: Projet Informatique de Cartographie Continue (PICC), Service Public de Wallonie, http://geoportail.wallonie.be/home.html

Saaty T.L. (1980). The Analytical Hierarchy Process. New York: MacGraw Hill.

Snook, B. (2004). Individual differences in distance travelled by serial burglars, Journal of Investigative Psychology and Offender Profiling, 1(1), p. 53-66.

Sevtsuk, A., Mekonnen, M. (2012). Urban Network Analysis Toolbox, International Journal of Geomatics and Spatial Analysis, 22/2, p. 287-305.

Trotta M., Donnay, J.-P. (2015). Étude du positionnement des caméras ANPR en Province de Liège - Scénarios 1 et 2, Rapports de recherche établis à la demande du Gouverneur de la Province de Liège, Unité de Géomatique de l'Université de Liège, p. $26 \& 13$.

Vandeviver C., Neutens T., van Daele S., Geurts D., Vander Beken T. (2015). A discrete spatial choice model of burglary target selection at the house-level, Applied Geography, 64, p. 24-34. 